\title{
Percepción de los Beneficios de la Tenencia de Animales de Compañía en Personas con Problemas de Infertilidad
}

\author{
Perception of the Benefits of the OWnership of Companion Animals in \\ People with Fertility Disorders
}

\author{
Beatriz Hugues H. ${ }^{1,2}$, Aimee Álvarez A. ${ }^{1}$, Lizet Castelo E.C. ${ }^{1}$, Loraine Ledón L. ${ }^{1}$,

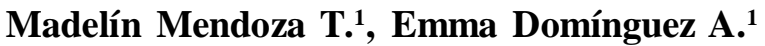

\section{Resumen}

El estudio tuvo como objetivo conocer la percepción que tienen las personas que padecen de infertilidad, con respecto a la tenencia de animales de compañía y su relación con la satisfacción personal y los beneficios sobre la salud psicoemocional. Se realizó una encuesta con base a un cuestionario autoadministrado. El 50 y $20 \%$ de las personas poseían perros y gatos, respectivamente. El $88 \%$ de los encuestados se sentían muy satisfechos por convivir con animales de compañía. El 92\% de los encuestados indicó que sus animales les proporcionaban compañía y bienestar, el $87 \%$ señaló que los liberaban de las tensiones y el $85 \%$ sentía que los estimulaban a cuidar más de su salud. Se concluye que el sector evaluado percibía que la tenencia de animales de compañía les ejerció efectos beneficiosos para su satisfacción personal y salud emocional.

Palabras clave: animales de compañía; infertilidad

\section{Abstract}

The aim of this study was to evaluate the perception of persons with fertility disorders regarding pet ownership and its relationship with personal satisfaction and benefits on psicoemotional health. A survey using a questionnaire was conducted. The results showed that 50 and $20 \%$ of the persons had dogs and cats as pets respectively. The $88 \%$ of persons were very satisfied living with pets. Also, $92 \%$ pointed out that their pets provided

\footnotetext{
${ }^{1}$ Instituto Nacional de Endocrinología (INEN), La Habana, Cuba
}

${ }^{2}$ E-mail: bettymig@infomed.sld.cu

Recibido: 6 de junio de 2014

Aceptado para publicación: 2 de noviembre de 2014 
company and well-being, $87 \%$ indicated that felt free of tensions, and $85 \%$ mentioned that pets constituted a motive to take better care of themselves. It is concluded that the studied sector perceived personal satisfaction and better emotional health due to pet ownership.

Key words: pets; infertility

\section{INTRODUCCIÓN}

Los animales de compañía contribuyen a prevenir o recuperar la salud de los seres humanos contra la presentación de enfermedades cardiovasculares, metabólicas, endocrinas (disminución de la frecuencia cardiaca, respiratoria, tensión arterial, niveles de colesterol y triglicéridos) (Wood et al., 2005) y mentales (reducción del estrés, angustias, depresiones y temores, promover la relajación, distracción) (Martínez, 2008). Como consecuencia, se puede mejorar la calidad de vida de las personas con las que se relacionan (Gómez et al., 2007; Mc Connell et al., 2011), lo cual puede conducir a mayores expectativas de longevidad y menor morbilidad en estas personas, preservando el equilibrio físico y mental (Mentzel, 2004).

La tenencia de animales facilita, además, la relación y socialización entre personas desconocidas, haciéndolas más solidarias y comunicativas; la comunicación entre generaciones por ser un foco de interés común, tanto en el seno de la familia como fuera de ella; y la participación en actividades recreativas (Jofré, 2005).

La infertilidad constituye un padecimiento que tiene importantes implicaciones en la vida social y psicológica de las personas que la sufren, pues acarrea una serie de dificultades en el seno de la familia que está en construcción, toda vez que, para una pareja, tener hijos significa haber llegado a la cúspide de sus expectativas (Calero et al., 2003). Calero y Santana (2006) refieren que la infertili- dad, por el sistema biomédico, se define como la incapacidad de una pareja para lograr un embarazo durante varios meses de actividad sexual sin la utilización de métodos anticonceptivos. Más que un problema médico, es un problema social de la salud reproductiva.

Estudios sobre aspectos emocionales de la parejas que solicitan atención médica por infertilidad muestran que estas padecen de sentimientos de inadecuación, desesperación, pena, envidia y celos hacia la mujer embarazada, miedo, pérdida de la libido, impotencia, inestabilidad emocional, pérdida de la autoestima, culpa, depresión e ideación suicida (Palacios, 2000; Llavona, 2008).

Teniendo en cuenta los efectos positivos que sobre la salud humana ejerce la convivencia con los animales de compañía, sobre todo por los efectos psicoemocionales y socializadores, podría considerarse que las personas con infertilidad se beneficiarían al convivir con esta clase de animales. No obstante, no se ha encontrado referencias en la literatura científica sobre los posibles beneficios de la tenencia de animales de compañía en personas que padecen de infertilidad en las condiciones de Cuba.

Sobre estas bases, el presente estudio tuvo como objetivo conocer la percepción que tienen las personas que padecen de infertilidad (y que atienden a sus animales de compañía en una clínica veterinaria) con respecto a los beneficios de la tenencia de animales de compañía, su relación con la satisfacción personal y la salud psicoemocional que les proporciona. 


\section{Materiales y Métodos}

Se realizó un estudio descriptivo, transversal, utilizando metodología cuantitativa de investigación, durante los meses de septiembre de 2011 a mayo de 2012. La población objeto de estudio estuvo constituida por 60 personas con infertilidad (33 mujeres y 27 hombres), las cuales asistieron con sus animales de compañía a solicitar los servicios de la consulta de oftalmología de la clínica principal de animales afectivos de La Habana «José Luis Callejas».

Se consideraron infértiles a las personas que manifestaron que tras 12 meses de actividad sexual sin tomar medidas anticonceptivas no habían logrado concebir y habían sido tratadas en consultas de salud reproductiva. En este grupo, como criterios de inclusión se consideraron hombres y mujeres, mayores de 18 años, que tuvieran a su cargo animales de compañía, y como criterios de exclusión a personas con condiciones físicas o de salud mental que les dificultaban la lectura y comprensión del cuestionario del estudio.

Se trabajó con un cuestionario autoadministrado sobre tenencia de animales de compañía de 10 ítems, en el cual se incluyeron los siguientes aspectos:

- Datos demográficos de las personas: sexo, edad, ocupación. Se registraba si acudían solas o acompañadas por sus parejas a la consulta, y si convivían solas o con otras personas.

- Satisfacción personal sobre la tenencia de animales de compañía: Valoración de la satisfacción que siente una persona al estar en contacto directo con un animal de compañía, así como el bienestar que le produce esta interrelación. Se exploró sobre la especie de animal de compañía que poseían y el tiempo de convivencia con ellos; además, se indagó sobre el animal preferido. Los encuestados podían seleccionar más de una respuesta.
- Beneficios sobre la salud psicoemocional: Valoración de los posibles efectos beneficiosos que ejerce la tenencia de animales de compañía, relacionados con los aspectos de la salud psicoemocional y la calidad de vida de las personas a su cargo.

La participación de los sujetos en el estudio fue voluntaria y anónima. A cada persona que cumplió con los criterios de inclusión se le solicitó su participación en el estudio, previa información de las características del estudio. Al consentir su participación, se le brindó el cuestionario. Toda la información recogida se manejó de forma confidencial. No se recolectaron datos de identidad personal y los datos obtenidos fueron codificados y analizados mediante estadística descriptiva en números enteros.

\section{Resultados y Discusión}

\section{Datos Demográficos}

Los datos demográficos de las personas objeto de estudio se presentan en el Cuadro 1. Las personas con problemas de infertilidad más asiduas a la clínica de animales de compañía fueron mujeres (55\%), y dentro de los grupos etarios, el mayor número estuvo comprendido entre los 40 y 49 años (52\%). Asimismo, el $84 \%$ mantenía algún tipo de vínculo laboral, y ninguno de los encuestados era estudiante.

Beovides et al. (2011) realizaron una evaluación de las personas que estaban al cuidado de animales de compañía en Santa Clara, Cuba, encontrando que los hombres eran en mayor número (53.3\%), y dentro de los grupos etarios sobresalían los de 18-30 años $(42.7 \%)$ y $31-50$ años $(26.7 \%)$; hallazgos diferentes a los del presente estudio.

La mayoría asistió a las consultas acompañada de sus parejas (85\%, Cuadro 1). Así mismo, estas personas se referían a los animales como a hijos o familiares cercanos y 
Cuadro 1. Datos demográficos (\%) de las personas con problemas de infertilidad y que tienen animales de compañía en La Habana, Cuba

\begin{tabular}{llccc}
\hline \multirow{2}{*}{ Variable } & & \multicolumn{2}{c}{ Género } & Total \\
\cline { 3 - 4 } & & $\begin{array}{c}\text { Mujeres } \\
(\mathrm{n}=33)\end{array}$ & $\begin{array}{c}\text { Hombres } \\
(\mathrm{n}=27)\end{array}$ & $\begin{array}{c}\text { (n=60) } \\
\text { Edad (años) }\end{array}$ \\
& $30-39$ & 39 & 30 & 35 \\
& $40-49$ & 52 & 52 & 52 \\
Ocupación & 50 o más & 9 & 19 & 13 \\
& Estudiantes & - & - & - \\
Acuden a la consulta & Trabajadores & 85 & 89 & 86 \\
& Sin vínculo laboral & 15 & 11 & 14 \\
Conviven con otras & Solos (n=28) & 21 & 8 & 15 \\
personas & En pareja (n=24) & 79 & 92 & 85 \\
\hline
\end{tabular}

queridos. Este comportamiento resulta muy interesante y muestra la estima y significado que tiene la posesión de los animales.

En un estudio realizado en Cuba se encontró, asimismo, la preferencia por perros y gatos (24\%, por especie) en comparación con otras especies (Beovides et al., 2011). En forma similar, en un estudio reciente en las provincias de Mayabeque y La Habana se obtuvo que los perros también fueron los preferidos $(60 \%)$ (L. Perdomo, datos no publicados).

Trabajos llevados a cabo en el Perú, en familias con niños y jóvenes se halló una mayor proporción de perros y gatos, seguidos por aves ornamentales, roedores, tortugas y animales exóticos como chimpancés y zorros (Alva et al., 2010; Arévalo et al., 2010), lo cual se corresponde a los hallazgos del presente estudio, excepto en la tenencia de animales exóticos.
Las respuestas sobre la satisfacción por la tenencia de animales de compañía fueron muy favorables. El 88\% indicó que estaba muy satisfecho de compartir su vida en el hogar con estos animales (Cuadro 3). Asimismo, se obtuvieron respuestas muy positivas sobre el efecto en la salud psicoemocional, y no se recibieron respuestas negativas. En general, los encuestados estimaron que sus animales les proporcionaban compañía y bienestar (92\% en cada caso), los liberaba de tensiones (87\%) y los estimulaban a cuidar más de su salud (85\%). Las mujeres de más de 50 años opinaron, sobre todo, que los animales constituían un compañero, mientras que para los hombres de este grupo etario constituían un medio de comunicación familiar.

En el estudio de Perdomo (datos no publicados) en Cuba, el 69\% de los entrevistados consideraron que era una necesidad poseer animales de compañía en las casas, por- 
Cuadro 2. Frecuencia (\%) de tenencia y preferencia de animales de compañía por personas con problemas de infertilidad en La Habana, Cuba

\begin{tabular}{lcccccc}
\hline \multirow{2}{*}{$\begin{array}{l}\text { Animal de } \\
\text { compañía }\end{array}$} & \multicolumn{3}{c}{ Animal de tenencia } & \multicolumn{3}{c}{ Animal preferido } \\
\cline { 2 - 7 } & $\begin{array}{c}\text { Mujeres } \\
(\mathrm{n}=33)\end{array}$ & $\begin{array}{c}\text { Hombres } \\
(\mathrm{n}=27)\end{array}$ & $\begin{array}{c}\text { Total } \\
(\mathrm{n}=60)\end{array}$ & $\begin{array}{c}\text { Mujeres } \\
(\mathrm{n}=33)\end{array}$ & $\begin{array}{c}\text { Hombres } \\
(\mathrm{n}=27)\end{array}$ & $\begin{array}{c}\text { Total } \\
(\mathrm{n}=60)\end{array}$ \\
\hline Perro & 55 & 45 & 50 & 67 & 67 & 67 \\
Gato & 21 & 18 & 20 & 18 & 15 & 16 \\
Aves ornamentales & 3 & 15 & 8 & 3 & 11 & 7 \\
Peces ornamentales & 3 & 7 & 5 & 3 & 7 & 5 \\
Tortugas & 3 & 4 & 3 & 3 & - & 2 \\
Roedores & 6 & 4 & 5 & 6 & - & 3 \\
Otros & - & - & - & - & - & - \\
Varias especies & 9 & 7 & 9 & & & \\
\hline
\end{tabular}

Cuadro 3. Tiempo de tenencia, satisfacción y efecto de la relación dueño-animal, expresado en porcentaje, de personas con problemas de infertilidad en La Habana, Cuba

\begin{tabular}{lccc}
\hline Característica & $\begin{array}{c}\text { Mujeres } \\
(\mathrm{n}=33)\end{array}$ & $\begin{array}{c}\text { Hombres } \\
(\mathrm{n}=27)\end{array}$ & $\begin{array}{c}\text { Total } \\
(\mathrm{n}=60)\end{array}$ \\
\hline Tiempo de la tenencia (años) & & & \\
$>1$ & 3 & - & 2 \\
$1-3$ & - & 7 & 3 \\
$4-6$ & 6 & 18 & 12 \\
$7-9$ & 24 & 15 & 20 \\
$>9$ & 67 & 60 & 63 \\
Satisfacción con la tenencia & & & \\
Muy satisfecho & 91 & 85 & 88 \\
Satisfecho & 9 & 15 & 12 \\
Efecto de la relación con el animal & & & \\
Brinda compañía & 94 & 89 & 92 \\
Brinda bienestar & 91 & 93 & 92 \\
Libera tensiones y estrés & 91 & 81 & 87 \\
Satisface contacto & 82 & 67 & 75 \\
Mejora mi ánimo & 91 & 74 & 83 \\
Me comunico con él & 79 & 81 & 80 \\
Medio de comunicación familiar & 75 & 85 & 80 \\
Cuido mejor de mi salud & 88 & 81 & 85 \\
Compañero para mi soledad & 91 & 63 & 78 \\
\hline
\end{tabular}


Cuadro 4. Evaluación de algunos aspectos sobre los beneficios para la salud psicoemocional relacionados con la tenencia de animales de compañía en las personas con problemas de infertilidad en La Habana, Cuba

\begin{tabular}{lccc}
\hline Características & $\begin{array}{c}\text { Mujeres } \\
(\mathrm{n}=33)\end{array}$ & $\begin{array}{c}\text { Hombres } \\
(\mathrm{n}=27)\end{array}$ & $\begin{array}{c}\text { Total } \\
(\mathrm{n}=60)\end{array}$ \\
\hline Beneficios relación dueño /animal & & & \\
$\quad$ Muy beneficiosa & 94 & 89 & 92 \\
Beneficiosa & 6 & 11 & 8 \\
Recomendar tenencia de animales & & & \\
Sí & 79 & 85 & 82 \\
No & 21 & 15 & 18 \\
Animal de compañía/calidad de vida & & & \\
Sí & 100 & 100 & 100 \\
No & 0 & 0 & 0 \\
\hline
\end{tabular}

que estos brindan compañía y entretenimiento a las personas y, además, protegen el hogar.

\section{Salud Psicoemocional}

En el Cuadro 4 se muestran los beneficios expresados por los encuestados en la salud psicoemocional debido a la tenencia de animales de compañía. La mayor parte de los encuestados expresaron que esta relación les resultaba muy beneficiosa (92\%). Asimismo, el $82 \%$ recomendaba la tenencia de animales de compañía a otras personas; sin embargo, las personas de mayor edad fueron las que dieron respuestas negativas, argumentando que debía tenerse en cuenta las condiciones y preferencias de las personas, ya que a todos no les gusta o no tienen condiciones para compartir su vida con mascotas.

Todas las personas indicaron, asimismo, que la tenencia de los animales de compañía les mejoraba su calidad de vida; respuesta de gran importancia pues indica el rol benéfico de estos animales en la vida de las personas. Por otro lado, señalaron la necesidad de fomentar planes educativos con respecto a la promoción sobre el cuidado y tenencia de los animales, así como para evitar su maltrato y abuso.

\section{Literatura Citada}

1. Alva R, Revilla E, Isla C. 2010. Convivencia con animales de compañía en niños y jóvenes estudiantes del distrito de Chiclayo, provincia de Chiclayo, Lambayeque. En: XXII Congreso Panamericano de Ciencias Veterinarias. Lima, Perú.

2. Arévalo W, Rachumi V, Eslava J. 2010. Tenencia responsable de animales de compañía en niños y jóvenes estudiantes del distrito de Ferreñafe, Lambayeque. En: XXII Congreso Panamericano de Ciencias Veterinarias. Lima, Perú.

3. Beovides LM, Silveira PE, Norman MO. 2011. Evaluación del cuidado de la salud de mascotas en el municipio Santa Clara, Cuba. En: VII Congreso Internacional de Ciencias Veterinarias. La Habana, Cuba. 
4. Calero JL, Magallanes M, Rechkemmer A, García M. 2003. Infertilidad o paternidad frustrada. Significados de este fenómeno desde la perspectiva masculina peruana. Rev Cubana Salud Públ 29 (Supl 1): 48-50.

5. Calero JL, Santana F. 2006. La infertilidad como evento de frustración personal. Reflexiones de un grupo de varones de parejas infértiles. Rev Cubana Endocrinol 17(1). [Internet]. Disponible en: http://scielo.sld.cu/scielo. php?pid= S1561-29532006000 100002\&script $=$ sci_arttext

6. Gómez LF, Aterhortua CG, Orozco SC. 2007. La influencia de las mascotas en la vida humana. Rev Col Cienc Pec 20: 377-386.

7. Jofré LM. 2005. Visita terapéutica de mascotas en hospitales. Rev Chil Infectol 22(3): 257-263. doi: 10.4067/S071610182005000300007

8. Llavona LM. 2008. El impacto psicológico de la infertilidad. Papeles del Psicólogo 29(2): 158-166.
9. Martínez A. 2008. La terapia asistida por animales: una nueva perspectiva y línea de investigación en la atención a la diversidad. Indivisa N. ${ }^{\circ}$ 9: 117-144.

10. McConnellAR, Brown CM, Shoda TM, Stayton LE, Martin CE. 2011. Friends with benefits: on the positive consequences of pet ownership. J Pers Soc Psychol 101: 1239-1252. doi: 10.1037/a0024506

11. Mentzel RE. 2004. Origen y evolución del vínculo humano-animal. Rev Med Vet 85(4): 139-145.

12. Palacios E. 2000. Aspectos emocionales en la infertilidad: una revisión de la literatura reciente. Rev Chil NeuroPsiquiat 38(2): 94-103. doi: 10.4067/ S0717-92272000000200004

13. Wood L, Giles-Corti B, Bulsara M. 2005. The pet connection: pets as a conduit for social capital? Soc Sci Med 61:1159-1173. doi: 10.1016/j.socscimed. 2005.01.017 